\title{
VALIDAÇÃO DE MÉTODO PARA DETERMINAÇÃo DE RESÍDUOS DE AMOXICILINA APLICADO À VALIDAÇÃO DE LIMPEZA EM INDÚSTRIA FARMACÊUTICA DE PENICILÂNICOS
}

\author{
Maria Luiza Pinheiro Costa Gomes e Scheilla Vitorino Carvalho de Souza* \\ Centro Universitário Newton Paiva, Rua Goitacazes, 1762, 30140-090 Belo Horizonte - MG, Brasil
}

Recebido em 22/7/09; aceito em 3/11/09; publicado na web em 24/3/10

\begin{abstract}
VALIDATION OF A METHOD FOR DETERMINATION OF AMOXICILLIN RESIDUES APPLIED TO CLEANING VALIDATION PROCESS IN PENICILLINS PHARMACEUTICAL INDUSTRY. The aim of this work was the single-laboratory validation of a quantitative method for the determination of amoxicillin residues in support of cleaning control and validation. Linearity was demonstrated between 2.5 and $17.5 \mu \mathrm{g} / \mathrm{mL}$, without matrix effects. Mean recoveries ranged from 84.00 to $103.74 \%$ and the relative standard deviation under repetitivity and within-reproducibility conditions were from 0.58 to $4.20 \%$ and from 0.79 to $4.39 \%$, respectively. The theoretical limits of detection and quantification were 0.133 and $0.442 \mu \mathrm{g} / \mathrm{mL}$, respectively. The studied method was suitable for cleaning control purpose within good manufacturing practices.
\end{abstract}

Keywords: amoxicillin; method validation; cleaning validation.

\section{INTRODUÇÃO}

A amoxicilina é um antibiótico $\beta$-lactâmico de espectro moderado. Trata-se de uma penicilina semissintética, derivada da ampicilina, a qual é inativada por $\beta$-lactamases, sendo então sensível a microorganismos produtores de penicilinases. É um composto estável em meio ácido, que foi projetado para uso oral. Atua por inibição da produção de proteínas da parede celular bacteriana, de modo geral, afetando a síntese de componentes dos peptideoglicanos da parede celular e causando lise osmótica. ${ }^{1}$

A fabricação de medicamentos requer cuidados específicos que visam tanto o atendimento das necessidades e expectativas dos pacientes, quanto de requisitos regulamentares. As Boas Práticas de Fabricação (BPF) direcionam a produção dos medicamentos para que qualidade e segurança sejam asseguradas. ${ }^{2}$ No contexto das BPF, atenção especial é dada à validação de processos, de procedimentos de limpeza e de métodos analíticos de controle. ${ }^{3}$

A validação da limpeza consiste em uma sistemática utilizada para assegurar que os procedimentos de limpeza de equipamentos removam, efetivamente, os resíduos existentes até um nível de aceitação pré-determinado, considerado seguro. ${ }^{4}$

Devido aos avanços das técnicas analíticas instrumentais, resíduos provenientes de processos de produção e de limpeza podem ser detectados em nível de traços. Contudo, a não detecção de resíduos de um provável contaminante não significa a inexistência de tais resíduos após o procedimento de limpeza, mas sim que não há resíduos presentes em níveis maiores que o limite de detecção do método utilizado. Desta forma, os métodos utilizados para analisar as amostras oriundas da limpeza dos equipamentos de produção dos medicamentos devem ser validados pela determinação de parâmetros de desempenho aplicáveis como linearidade, seletividade, exatidão, precisão e limites de detecção e quantificação. Tais processos são estruturados em conformidade com regulamentos, como o Guia para Validação de Métodos Analíticos e Bioanalíticos da Agência Nacional de Vigilância Sanitária (ANVISA), ${ }^{5}$ e protocolos internacionais, como o Guia Harmonizado pela Association of Official Analytical Chemists (AOAC International), International Standards Organization (ISO) e International Union of Pure and Applied Chemistry (IUPAC). ${ }^{6} \mathrm{~A}$ validação de métodos ana-

\footnotetext{
*e-mail: scheillavitorino@terra.com.br
}

líticos constitui, assim, um requisito fundamental para os processos de validação de limpeza, pois avalia a adequação do método utilizado para a quantificação dos resíduos do fármaco, nos níveis de concentração próximos aos critérios de aceitação estabelecidos.

As contaminações cruzadas por penicilinas são tratadas como críticas pelo United States Food and Drug Administration (FDA), devido ao potencial alergênico destes compostos, ${ }^{2,4}$ pois medicamentos contaminados por fármacos de elevada alergenicidade podem causar efeitos colaterais inesperados. Assim, anafilaxia pode ser induzida, inesperadamente, pela administração de medicamentos não penicilânicos que estejam contaminados com estes compostos. ${ }^{7}$

Considerando, então, que o controle de resíduos críticos, como a amoxicilina, depende da confiabilidade do método analítico empregado, o presente trabalho objetivou validar o método de cromatografia líquida de alta eficiência com detecção ultravioleta (CLAE-UV), previsto na Farmacopeia Americana, ${ }^{8}$ para a determinação de resíduos de amoxicilina, oriundos do processamento de pó para suspensão oral, visando a validação do processo de limpeza em uma indústria farmacêutica de penicilânicos.

\section{PARTE EXPERIMENTAL}

\section{Soluções}

As soluções foram preparadas no momento das análises e utilizadas até $6 \mathrm{~h}$ após o preparo.

\section{Solução estoque de amoxicilina $(0,25 \mathrm{mg} / \mathrm{mL})$}

$\mathrm{O}$ equivalente a $25,0 \mathrm{mg}$ de amoxicilina anidra foram pesados do padrão de amoxicilina (tri-hidratada $88 \%$ padronizada frente ao padrão USP lote JOC 043 86,2\%), em balança analítica (Ohaus Adventurer OHUS) e transferidos, quantitativamente, para balão volumétrico de $100 \mathrm{~mL}$ (Pyrex). Em seguida, $70 \mathrm{~mL}$ de tampão fosfato de potássio $\mathrm{pH}$ 5,0 foram adicionados e a solução foi levada ao ultrassom (Branson $3510 \mathrm{R}-\mathrm{MT}$ ) por $20 \mathrm{~min}$. O volume foi completado com tampão fosfato de potássio pH 5,0 e a solução homogeneizada.

Curva de calibração de amoxicilina $(2,5$ a $17,5 \mu \mathrm{g} / \mathrm{mL})$

Alíquotas de 0, 100, 200, 300, 400, 500, 600 e $700 \mu \mathrm{L}$ da solução estoque de amoxicilina foram pipetadas (pipetador automático 
de 100 a $1000 \mu \mathrm{L}$, Brand Tranferpette) e transferidas para um balão volumétrico de $10 \mathrm{~mL}$ (Pyrex), para diluição com tampão fosfato de potássio $\mathrm{pH}$ 5,0. As soluções foram homogeneizadas e filtradas em membrana de 0,45 $\mu$ m (Sartorius Stedim Biotech).

Solução estoque de placebo (excipientes da formulação do pó para suspensão oral de amoxicilina $125 \mathrm{mg} / 5 \mathrm{~mL}$ sem o princípio ativo)

Uma quantidade correspondente a 140,6 mg de placebo do pó para suspensão oral de amoxicilina foi pesada em balança analítica (Ohaus Adventurer OHUS) e transferida, quantitativamente, para balão volumétrico de $100 \mathrm{~mL}$ (Pyrex). Em seguida, $70 \mathrm{~mL}$ de tampão fosfato de potássio $\mathrm{pH}$ 5,0 foram adicionados e a solução foi levada ao ultrassom (Branson $3510 \mathrm{R}-\mathrm{MT}$ ) por $20 \mathrm{~min}$. O volume foi completado com tampão fosfato de potássio pH 5,0 e a solução homogeneizada.

\section{Curva de calibração de amoxicilina matrizada $(2,5$ a $17,5 \mu \mathrm{g} / \mathrm{mL})$}

Alíquotas de 0,100, 200, 300, 400, 500, 600 e $700 \mu \mathrm{L}$ da solução estoque de amoxicilina foram pipetadas (pipetador automático de 100 a $1000 \mu \mathrm{L}$, Brand Tranferpette) e transferidas para um balão volumétrico de $10 \mathrm{~mL}$ (Pyrex). Adicionou-se também $1 \mathrm{~mL}$ da solução estoque de placebo a cada uma das diluições e o volume foi completado com tampão fosfato de potássio pH 5,0. As soluções foram homogeneizadas e filtradas em membrana de 0,45 $\mu \mathrm{m}$ (Sartorius Stedim Biotech). A concentração final de placebo nas soluções da curva $(140,6 \mu \mathrm{g} / \mathrm{mL})$ correspondeu ao teor de placebo esperado para resíduos de amoxicilina no nível de aceitação de $10 \mu \mathrm{g} / \mathrm{mL}$, consideradas as proporções da formulação do pó para suspensão oral.

\section{Amostragem}

$\mathrm{Na}$ indústria onde os experimentos foram realizados, a amostragem direta de superfície foi realizada com o auxílio de swab (Clean Tips, Texwipe TX 761 Alpha Swab) e gabaritos de aço inoxidável $304 \mathrm{com} 25 \mathrm{~cm}^{2}$. Assim, o swab foi passado na área de $25 \mathrm{~cm}^{2}$, cujo limite máximo de aceitação para o ativo amoxicilina foi de $10 \mu \mathrm{g} /$ $\mathrm{cm}^{2}$. Este critério foi estabelecido levando em consideração a DL50 da amoxicilina, o tamanho de lote e também a área de contato do ativo com equipamentos e utensílios durante o processo produtivo. ${ }^{9}$

O swab foi colocado em um tubo de ensaio (Pyrex) e diluído com $25 \mathrm{~mL}$ de tampão fosfato de potássio pH 5,0 (resultando em uma concentração final de $10 \mu \mathrm{g} / \mathrm{mL}$, caso o teor de amoxicilina corresponda ao limite de aceitação). O tubo de ensaio foi agitado em agitador tipo Vortex (Phoenix AP56), por $1 \mathrm{~min}$, na velocidade máxima. O tubo foi levado para ultrassom (Branson 3510 R-MT) por mais 30 min para extração da amoxicilina.

\section{Procedimento analítico}

O método empregado neste trabalho para quantificação do resíduo do ativo foi por CLAE-UV, conforme descrito na Farmacopeia Americana. $^{8}$

\section{Preparo da amostra}

Uma alíquota foi tomada do tubo de ensaio (Pyrex) contendo a solução proveniente do $s w a b$ e filtrada em membrana de $0,45 \mu \mathrm{m}$ (Sartorius Stedim Biotech), diretamente para um frasco de $2 \mathrm{~mL}$.

As amostras utilizadas para validação analítica, nos testes de exatidão e precisão, foram preparadas conforme as alíquotas da curva matrizada, sendo utilizadas a menor, média e maior concentração da curva.

\section{Determinação por CLAE-UV}

O cromatógrafo líquido de alta eficiência Schimadzu Série 10 era composto pelos seguintes módulos: bomba isocrática LC-10 ATVP, injetor manual SIL-10 AF, detector SPD-10 AVP, sistema de aquisição de dados e integrador SCL-10 AVP.

A análise cromatográfica foi conduzida para as preparações nas condições descritas a seguir: volume de injeção de $50 \mu \mathrm{L}$; temperatura do forno de $25^{\circ} \mathrm{C}$; coluna $\mathrm{C}_{18} 250$ x 4,6 mm, $5 \mu \mathrm{m}$ (17046 Phenomenex luna); fase móvel composta por tampão fosfato de potássio pH 5,0:acetonitrila grau cromatográfico (JTBaker, USA) (96:4, v/v); sistema isocrático; fluxo de $1,5 \mathrm{~mL} / \mathrm{min}$; detector $\mathrm{UV}$; comprimento de onda de $230 \mathrm{~nm}$; tempo total de corrida de $3 \mathrm{~min}$; integração pelo parâmetro da área dos picos.

\section{Procedimento de validação}

Os parâmetros linearidade, efeitos de matriz, seletividade, exatidão, precisão e limites de detecção e de quantificação foram estabelecidos em ensaios com soluções padrão e formulações. A adequação para uso do método foi avaliada em função dos parâmetros estudados e respectivos critérios de aceitabilidade definidos. O procedimento de validação adotado foi baseado em um delineamento experimental simples e testes estatísticos robustos, incluindo verificações das premissas relacionadas a cada teste. ${ }^{6,10} \mathrm{O}$ nível de significância adotado nos testes de hipóteses foi $\alpha=0,05$.

\section{Linearidade e seletividade (efeitos de matriz)}

A avaliação da linearidade foi conduzida conforme procedimentos propostos por Souza e Junqueira. ${ }^{11}$ Uma curva de calibração foi preparada, nos níveis de concentração 2,5; 5,1; 7,6; 10,2; 12,7; 15,3 e $17,8 \mu \mathrm{g} / \mathrm{mL}$ de amoxicilina em tampão fosfato de potássio $\mathrm{pH}$ 5,0 (correspondentes a 2,5 a 17,8 $\mu \mathrm{g} / \mathrm{mL}$ de amoxicilina em solução de $s w a b)$, sendo feitas três replicatas independentes de cada nível.

$\mathrm{Na}$ verificação dos efeitos de matriz, uma segunda curva de calibração foi estudada nos níveis de 2,5; 5,0; 7,5; 10,0; 12,5; 15,0 e $17,6 \mu \mathrm{g} / \mathrm{mL}$ de amoxicilina em matriz (placebo do pó para suspensão), sendo cada nível em três replicatas independentes. Para simulação dos interferentes de matriz, placebo do pó para suspensão foi adicionado a cada solução de forma a fornecer uma concentração final do placebo correspondente ao teor de $10,0 \mu \mathrm{g} / \mathrm{mL}$ de amoxicilina, consideradas as proporções da formulação.

As soluções das duas curvas, usual e de matriz, foram analisadas em ordem aleatória. Brancos foram preparados em triplicata para avaliação do ruído, mas não foram incluídos nos cálculos da regressão.

Os parâmetros da regressão foram estimados pelo método dos mínimos quadrados ordinários (MMQO). Em seguida, gráficos dos resíduos da regressão foram construídos e examinados para investigação de perfis óbvios, sendo indicados como outliers (valores dispersos) os pontos fora do intervalo $\pm t_{(0,975 ; n-2)} s_{\text {res }}$, sendo $s_{\text {res }}$ o desvio padrão dos resíduos da regressão. ${ }^{12}$ Os outliers foram formalmente diagnosticados pelo teste de resíduos padronizados Jacknife. ${ }^{13}$ Este teste foi aplicado sucessivamente até que novos outliers não fossem detectados ou até uma exclusão máxima de $22,2 \%$ no número original de resultados. ${ }^{14}$ As premissas relativas à análise de regressão foram verificadas sendo: normalidade,,$^{15}$ homoscedasticidade ${ }^{16,17} \mathrm{e}$ independência dos resíduos da regressão. ${ }^{18}$ Testes de F foram conduzidos para verificar o ajuste ao modelo linear por meio da avaliação das significâncias da regressão e do desvio da linearidade. ${ }^{19}$

Uma vez comprovadas as premissas e o ajuste ao modelo linear, a inclinação e a interseção obtidas para a curva usual foram comparadas com aquelas estimadas para a curva em matriz. A premissa de homogeneidade das variâncias dos resíduos das curvas foi avaliada pelo teste de $\mathrm{F}^{20}$ para verificar se inclinações e interseções seriam comparadas pelo teste de $\mathrm{t}$ com as variâncias combinadas ou distintas. ${ }^{21}$ 


\section{Exatidão, precisão e limites}

Devido à inexistência de materiais de referência certificados devidamente caracterizados para amoxicilina, os parâmetros recuperação e precisão (sob condições de repetitividade e reprodutibilidade parcial) foram pesquisados em ensaios com formulações em três níveis de concentração, 2,5;10,0 e 17,5 $\mu \mathrm{g} / \mathrm{mL}$, sendo seis replicatas independentes de cada nível. Tais amostras foram preparadas conforme as alíquotas da curva matrizada. Em seguida, as replicatas de cada nível foram divididas em dois lotes, com três replicatas cada, os quais foram analisados em dois diferentes ensaios, por dois analistas.

Recuperação e precisão, considerando a etapa de amostragem, também foram avaliadas. Uma solução padrão matrizada de amoxicilina foi adicionada aos gabaritos de aço inoxidável $304 \mathrm{de} 25 \mathrm{~cm}^{2}$, para obtenção do nível correspondente ao limite máximo de aceitação de $10 \mu \mathrm{g} / \mathrm{cm}^{2}$, ou seja, $250 \mu \mathrm{g}$ de amoxicilina. Após secagem da solução adicionada, o procedimento de amostragem foi realizado e as amostras encaminhadas para análise. Vinte e quatro replicatas foram estudadas, as quais foram divididas em quatro lotes, com seis replicatas cada, que foram analisados em quatro diferentes ensaios, por dois analistas.

As concentrações de amoxicilina nas amostras foram preditas por meio de equações obtidas de curvas de calibração usuais, devido aos resultados dos estudos de efeitos de matriz.

Os resultados de recuperação obtidos foram analisados quanto à presença de outliers pelo teste de Grubbs. ${ }^{22-24}$ As estatísticas de Grubbs para um outlier $\left(G_{1}\right)$, dois outliers vicinais $\left(G_{2}\right)$ e dois outliers polares $\left(G_{3}\right)$ foram calculadas simultaneamente para os resultados obtidos no nível de concentração estudado. A indicação de outlier por, pelo menos, uma destas estatísticas seria suficiente para excluir o dado. Após um primeiro ciclo, o teste de Grubbs foi sucessivamente aplicado até que novos outliers não fossem detectados ou até uma exclusão máxima de $22,2 \%$ no número original de resultados de recuperação aparente. ${ }^{14}$

A inexatidão foi investigada por meio da média de recuperação obtida para as seis replicatas de amostras adicionadas nos níveis de concentração estudados, após tratamento de outliers. O critério de aceitabilidade adotado foi de média de recuperação 80 e $115 \%$, conforme previsto pela AOAC Internationa $l^{25}$ para analitos em concentrações de $10 \mu \mathrm{g} / \mathrm{mL}$.

As precisões sob condições de repetitividade e reprodutibilidade parcial foram expressas em termos de desvios padrão relativos e estimadas por análise de variância dos resultados de recuperação obtidos para as seis replicatas de cada nível de concentração estudado. ${ }^{26-29}$ As premissas para análise de variância, normalidade ${ }^{15} \mathrm{e}$ homoscedasticidade dos resíduos dos resultados de recuperação, ${ }^{16,17}$ foram previamente testadas. Segundo a ANVISA, ${ }^{5}$ o valor máximo aceitável para desvios padrão relativos deve ser definido de acordo com o método, concentração do analito, tipo de matriz e finalidade do método, não se admitindo valores superiores a 5\%. Contudo, considerando-se que a precisão varia com a concentração do analito, os valores de desvios padrão relativos de reprodutibilidade parcial $\left(\mathrm{DPR}_{\mathrm{R}}\right)$ foram comparados com aqueles estimados pelas equações de Horwitz. ${ }^{30}$ Esta função constitui uma generalização útil e amplamente reconhecida do desvio padrão sob condições de reprodutibilidade. Por isto, valores estimados a partir desta equação são muitas vezes utilizados como requisitos analíticos para precisão. Para concentrações de analitos entre 120 ppb e 13,8\%, a função corresponde a $\sigma_{H}=0,02 \mathrm{c} / m r$, onde $\mathrm{c}=$ concentração teórica adicionada do analito expressa como fração de massa (por exemplo, $10^{-6}$ para $1 \mu \mathrm{g} / \mathrm{mL}$ ou $1 \mathrm{ppm}$ ), $\mathrm{mr}=$ fração de massa (por exemplo $10^{-6} \mathrm{para} \mu \mathrm{g} / \mathrm{mL}$ ou ppm), $\sigma_{H}=$ desvio padrão de reprodutibilidade estimado. Como critério para avaliação dos valores de desvios padrão relativos de repetitividade $\left(\mathrm{DPR}_{\mathrm{r}}\right)$, foi considerado o critério de dois terços o valor do $\mathrm{DPR}_{\mathrm{R}}{ }^{30}$

Os limites de detecção e de quantificação teóricos foram estabelecidos utilizando-se o desvio padrão da interseção e a inclinação estimados para a curva matrizada. Sendo o limite de detecção es- tabelecido como três vezes o desvio padrão do intercepto dividido pela inclinação e o limite de quantificação como dez vezes o desvio padrão do intercepto dividido pela inclinação. ${ }^{5}$

\section{Aplicação do método validado}

Amostras provenientes de três diferentes lotes do produto, pó para suspensão oral, foram analisadas pelo método validado. Para cada lote, foram amostrados gabaritos de aço inoxidável $304 \mathrm{de} 25 \mathrm{~cm}^{2}$, os quais foram colocados em pontos críticos de equipamentos envolvidos no processo, sendo: cinco pontos da dosadora de pós (superfície interna do funil de alimentação, hélice de agitação, rosca dosadora, aresta da calha de escoamento e fresta do visor de escoamento) e três pontos do misturador (face interna da câmara de mistura na entrada do produto, face interna do bocal de saída do produto e encaixe da borracha de vedação).

\section{RESULTADOS E DISCUSSÃO}

\section{Linearidade}

Os resultados obtidos nos experimentos para avaliação da linearidade das curvas de amoxicilina usual e em matriz estão demonstrados nas Figuras 1 e 2 e na Tabela 1 . As premissas de que os resíduos da regressão seguem a distribuição normal, são homoscedásticos e independentes foram confirmadas, garantindo uma aplicação segura dos testes de hipóteses de t e F, além do emprego adequado do MMQO, sem ponderações.

A Figura 1 ilustra os gráficos exploratórios dos resíduos da regressão com indicação dos outliers tratados. Os perfis destes gráficos de resíduos demonstraram que não houve tendências óbvias que demonstrassem heteroscedasticidade ou desvio de linearidade. Os intervalos de confiança dos resíduos $\left( \pm t_{(0,975 ; n-2)} s_{\text {res }}\right)$ sugeriram a presença um outlier no nível $15,3 \mu \mathrm{g} / \mathrm{mL}$ da curva usual e três outliers nos níveis 12,$5 ; 15,0$ e 17,6 $\mu \mathrm{g} / \mathrm{mL}$ da curva em matriz, os quais foram confirmados pelo teste de resíduos padronizados Jacknife.
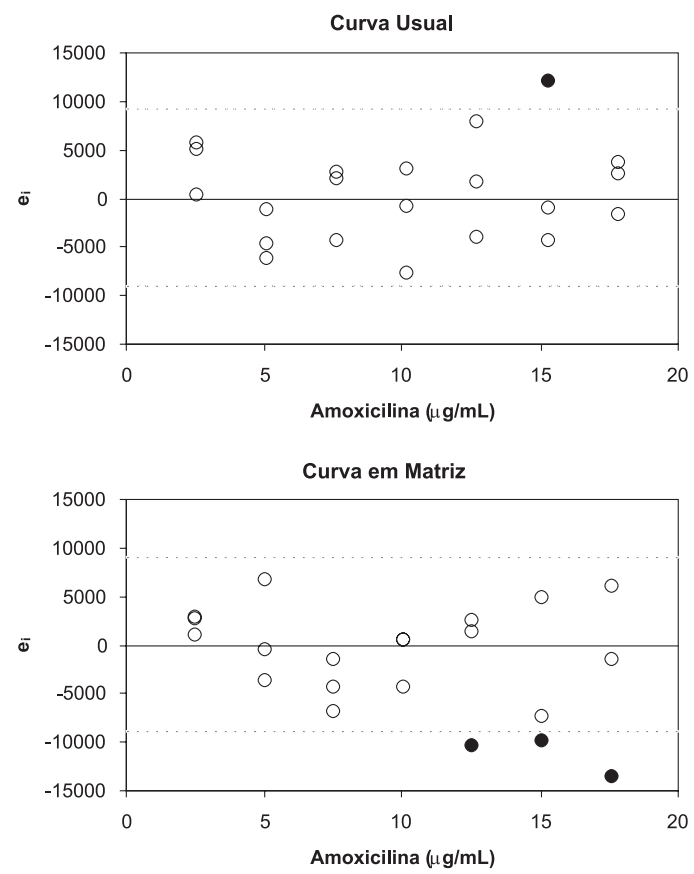

Figura 1. Gráficos exploratórios dos resíduos da regressão da curva usual de amoxicilina $(2,5$ a $17,8 \mu \mathrm{g} / \mathrm{mL})$ e da curva de amoxicilina em matriz (2,5 a 17,6 $\mu \mathrm{g} / \mathrm{mL})$, com indicação dos respectivos outliers diagnosticados pelo teste de resíduos padronizados Jacknife. $e_{i}=$ resíduo da regressão, $\bullet=$ outlier, - - - $= \pm t_{(0,975 ; n-2)} s_{\text {res }}$ 
O teste de Ryan-Joiner indicou correlação significativa entre os resíduos da regressão obtidos para cada curva e os valores normais teoricamente esperados. O coeficiente de correlação foi de 0,9914 (valor crítico de 0,9503) e de 0,9896 (valor crítico de 0,9461) para as curvas usual e em matriz, respectivamente, demonstrando que o desvio da distribuição normal não foi significativo $(p>0,10)$. A premissa de homoscedasticidade dos resíduos da regressão também foi confirmada. A variabilidade dos resíduos ao longo das concentrações estudadas foi constante, uma vez que as estatísticas t de Levene, estimadas em 0,310 para a curva usual (com t crítico de 2,101) e -0,394 para a curva em matriz (com t crítico de 2,120), não foram significativas $(p>0,05)$. Houve independência dos resíduos da regressão, uma vez que as estatísticas de Durbin-Watson calculadas para as curvas usual e em matriz sugeriram que não houve autocorrelação $(p>0,10)$. A regressão apresentou-se altamente significativa $(p<0,001)$, enquanto o desvio de linearidade não foi significativo $(p>0,05)$, indicando linearidade na faixa estudada (Tabela 1)

Tabela 1. Avaliação da linearidade para curvas de calibração de amoxicilina usual $(2,5$ a $17,8 \mu \mathrm{g} / \mathrm{mL})$ e em matriz $(2,5$ a $17,6 \mu \mathrm{g} / \mathrm{mL})$

\begin{tabular}{lcc}
\hline Estatística & Curva Usual & Curva em Matriz \\
\hline Número de observações & & \\
$n$ & 20 & 18 \\
Normalidade & & \\
$R$ & 0,9914 & 0,9896 \\
$p$ & $>0,10$ & $>0,10$ \\
Homoscedasticidade & & \\
$t_{L}$ & 0,310 & $-0,394$ \\
$p$ & $>0,05$ & $>0,05$ \\
Independência & & \\
$d$ & 1,696 & 2,473 \\
$p$ & $>0,10$ & $>0,10$ \\
Regressão & & \\
$F$ & $6,457 \times 10^{+4}$ & $5,534 \times 10^{+4}$ \\
$p$ & $1,88 \times 10^{-33}$ & $9,56 \times 10^{-30}$ \\
Desvio da linearidade & & \\
$F$ & 1,586 & 1,065 \\
$p$ & 0,23 & 0,43 \\
\hline
\end{tabular}

$n=$ número de observações, $R=$ coeficiente de correlação de Ryan-Joiner, $p$ $=$ significância, $t_{L}=$ estatística $t$ de Levene, $d=$ estatística de Durbin-Watson, $F=$ razão entre variâncias.

As curvas de calibração com respectivas equações e coeficientes de determinação estão ilustradas na Figura 2.

Estudos de validação de métodos aplicados ao controle de resíduos de $\beta$-lactâmicos e outros compostos, oriundos de processos de limpeza em indústrias farmacêuticas, abordaram a avaliação da linearidade tendo como base o valor do coeficiente de correlação, ${ }^{7,31,32}$ embora este não seja um parâmetro adequado para avaliação da linearidade. ${ }^{6,11}$

\section{Seletividade (efeitos de matriz)}

$\mathrm{O}$ teste de $\mathrm{F}$ indicou homogeneidade das variâncias dos resíduos das curvas usual e em matriz ( $F=1,054$, com $p=0,461)$ e o uso do teste de $t$ com variâncias combinadas para avaliação dos efeitos de matriz. Assim, não houve diferença significativa quando as interseções ( $t_{a}$ calculado de 0,971 , com $t$ crítico de 2,032$)$ e as inclinações ( $\mathrm{t}_{\mathrm{b}}$ calculado de 1,663, com t crítico de 2,032) da curva usual e em matriz foram comparadas entre si, indicando ausência de efeitos de matriz $(p>0,05)$ (Tabela 2). Com base nestes resultados, foi possível inferir que curvas usuais de amoxicilina forneceram as mesmas
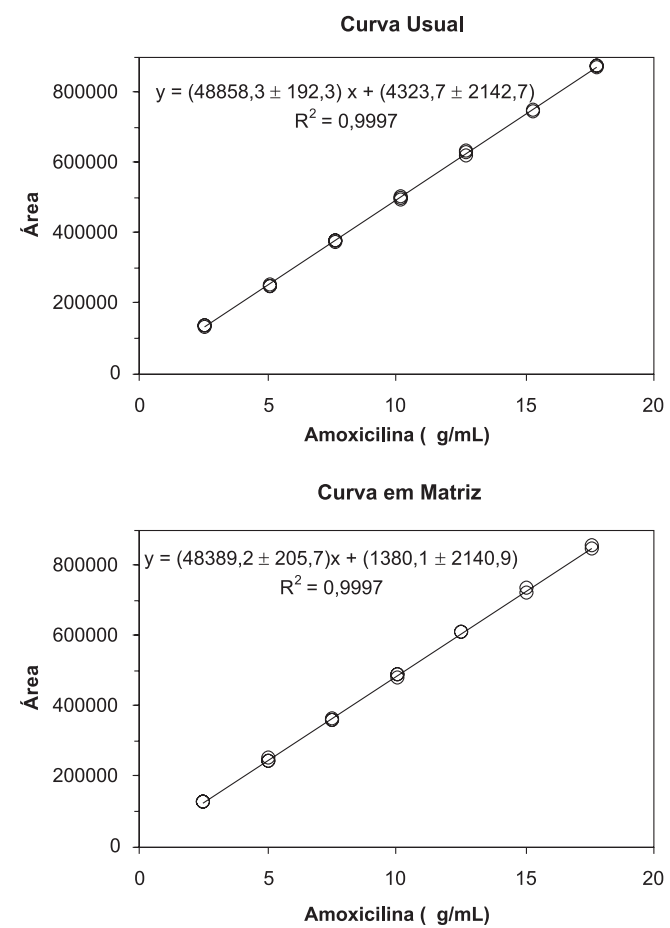

Figura 2. Curvas de calibração de amoxicilina usual (2,5 a 17,8 $\mu \mathrm{g} / \mathrm{mL}) e$ em matriz (2,5 a 17,6 $\mu \mathrm{g} / \mathrm{mL})$, com respectivas equações e coeficientes de determinação. Área $=$ resposta obtida de área dos picos, $y=$ resposta, $x=$ concentração de amoxicilina em $\mu \mathrm{g} / \mathrm{mL}, R^{2}=$ coeficiente de determinação

respostas que amostras de soluções de swabs oriundas de processos de produção de pó para suspensão oral, nas mesmas concentrações.

Tabela 2. Comparações entre as interseções e inclinações das curvas usual e em matriz para amoxicilina

\begin{tabular}{lc}
\hline Estatística & Comparação entre curva usual e curva em matriz \\
\hline & Interseção \\
$t_{a}$ & 0,971 \\
$p$ & 0,339 \\
& Inclinação \\
$t_{b}$ & 1,663 \\
$p$ & 0,106 \\
\hline$t_{a}=$ estatística t para contrastes entre interseções, $t_{b}=$ estatística $t$ para con- \\
trastes entre inclinações, $p=$ significância.
\end{tabular}

Na Figura 3 são apresentados cromatogramas obtidos para uma solução de amoxicilina da curva usual e para a solução de placebo da curva matrizada. Tais resultados indicaram a seletividade do método, confirmando a capacidade de determinar o analito em estudo na presença de compostos da matriz.

Alencar et ll $^{31}$ e Fekete et al. ${ }^{32}$ avaliaram a seletividade de métodos para validação de processos de limpeza por meio de experimentos com placebos. Fukutsu et ll $^{7}$ discutiram sobre a possibilidade de interferências nos resultados devido a compostos coexistentes, contudo, estes autores não verificaram a seletividade, alegando que não existiam outros compostos no processo além do $\beta$-lactâmico estudado. Nos trabalhos supracitados não foram conduzidos testes de efeitos de matriz como especificado no presente trabalho.

\section{Exatidão, precisão e limites}

O teste de Grubbs não indicou presença de outliers $(p>0,05)$ 
Solução de amoxicilina $2,5 \mu \mathrm{g} / \mathrm{mL}$

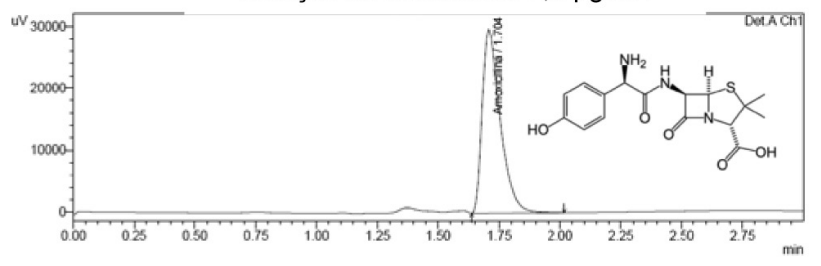

Solução de placebo

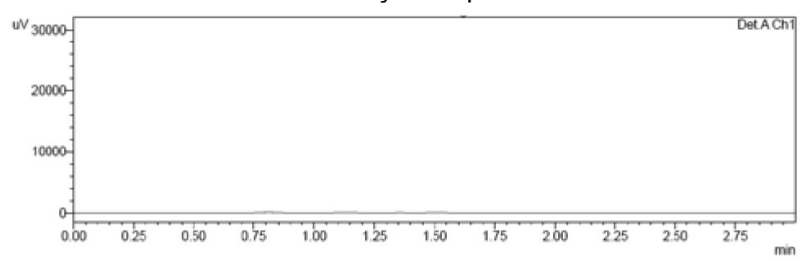

Figura 3. Cromatogramas obtidos para soluções de amoxicilina $(2,5 \mu \mathrm{g} /$ $m L$ ) da curva de calibração usual e para a solução de placebo do pó para suspensão oral da curva de calibração matrizada

entre os resultados de porcentagem de recuperação obtidos para as seis replicatas de amostras analisadas nos três níveis de concentração estudados e para as vinte e quatro replicatas de amostras analisadas no nível crítico de controle, incluindo a etapa de amostragem (Tabela 3).

Tabela 3. Estatísticas de Grubbs para verificação de outliers entre os resultados de porcentagem de recuperação, em cada nível de concentração estudado

\begin{tabular}{|c|c|c|c|c|c|c|}
\hline \multirow[t]{3}{*}{ Estatística } & \multicolumn{4}{|c|}{ Etapa de amostragem excluída } & \multicolumn{2}{|c|}{$\begin{array}{c}\text { Etapa de amostragem } \\
\text { incluída }\end{array}$} \\
\hline & \multirow{2}{*}{$\begin{array}{l}\text { Valor } \\
\text { crítico }\end{array}$} & \multicolumn{3}{|c|}{ Valor calculado } & \multirow{2}{*}{$\begin{array}{l}\text { Valor } \\
\text { crítico }\end{array}$} & \multirow{2}{*}{$\begin{array}{c}\text { Valor calculado } \\
10,0 \\
\mu \mathrm{g} / \mathrm{mL} \\
\end{array}$} \\
\hline & & $\begin{array}{c}2,5 \\
\mu \mathrm{g} / \mathrm{mL}\end{array}$ & $\begin{array}{c}10,0 \\
\mu \mathrm{g} / \mathrm{mL}\end{array}$ & $\begin{array}{c}17,5 \\
\mu \mathrm{g} / \mathrm{mL}\end{array}$ & & \\
\hline$G_{1 L}$ & 1,822 & 1,617 & 1,373 & 1,493 & 2,663 & 2,244 \\
\hline$G_{1 U}$ & 1,822 & 1,181 & 1,046 & 1,318 & 2,663 & 1,674 \\
\hline$G_{2}$ & 3,01 & 2,80 & 2,42 & 2,81 & 4,73 & 3,918 \\
\hline$G_{3 L}$ & 0,9436 & 0,8502 & 0,8771 & 0,8204 & 0,4505 & 0,264 \\
\hline$G_{3 U}$ & 0,9436 & 0,5719 & 0,5609 & 0,5782 & 0,4505 & 0,167 \\
\hline
\end{tabular}

$G_{1 L}=$ estatística de Grubbs para o menor valor; $G_{1 U}=$ estatística de Grubbs para o maior valor; $G_{2}=$ estatística de Grubbs para menor e maior valores; $G_{3 L}=$ estatística de Grubbs para dois menores valores; $G_{3 U}=$ estatística de Grubbs para dois maiores valores.

Os resíduos, obtidos pela diferença entre a recuperação média e os valores individuais de recuperação obtidos em cada dia, apresentaram distribuição normal $(p>0,10)$ e homoscedasticidade $(p>0,05)$ (Tabela 4), permitindo a estimativa de $\mathrm{DPR}_{\mathrm{r}}$ e $\mathrm{DPR}_{\mathrm{R}}$ por análise de variância.

Os valores individuais de recuperação variaram de 101,67 a 105,26\%, de 98,62 a 102,05\% e de 97,69 a 99,68\%, nos níveis 2,$5 ; 10,0$ e 17,5 $\mu \mathrm{g} /$ $\mathrm{mL}$, respectivamente. $\mathrm{O}$ perfil destes resultados de recuperação individuais nos dois diferentes ensaios pode ser observado na Figura 4. As médias de recuperação alcançadas para as seis replicatas de amostras foram de $103,74 \%$ para $2,5 \mu \mathrm{g} / \mathrm{mL}, 100,57 \%$ para $10,0 \mu \mathrm{g} / \mathrm{mL}$ e $98,75 \%$ para $17,5 \mu \mathrm{g} / \mathrm{mL}$, atendendo aos critérios estabelecidos de 80 a $115 \%{ }^{24}$ As porcentagens de recuperação individuais variaram de 75,80 a 90,12\%, com média de $84,00 \%$, nos experimentos com 24 amostras incluindo a etapa de amostragem de superfícies de um gabarito contendo resíduos de amoxicilina no limite máximo de aceitação de $10 \mu \mathrm{g} / \mathrm{cm}^{2}$. Estes resultados demonstraram que a recuperação do método é adequada e que o mesmo não é inexato nos níveis de estudados (Tabela 5).

Os valores de $\mathrm{DPR}_{\mathrm{r}}$ e $\mathrm{DPR}_{\mathrm{R}}$, estimados por análise de variância, para soluções de $s w a b$ sem inclusão da etapa de amostragem, variaram entre 0,72 e $1,19 \%$ e 0,93 e $1,51 \%$, respectivamente, na faixa de
Tabela 4. Avaliação das premissas para análise de variância (normalidade e homoscedasticidade dos resíduos dos resultados de recuperação de amoxicilina)

\begin{tabular}{|c|c|c|c|c|}
\hline \multirow{2}{*}{ Estatística } & \multicolumn{4}{|c|}{ Concentração $(\mu \mathrm{g} / \mathrm{mL})$} \\
\hline & 2,5 & 10,0 & 17,5 & $10,0^{*}$ \\
\hline \multicolumn{5}{|c|}{ Normalidade } \\
\hline$R$ & 0,9909 & 0,9580 & 0,9763 & 0,9773 \\
\hline$p$ & $\mathrm{p}>0,10$ & $\mathrm{p}>0,10$ & $\mathrm{p}>0,10$ & $\mathrm{p}>0,10$ \\
\hline \multicolumn{5}{|c|}{ Homoscedasticidade } \\
\hline$F_{L}$ & 0,560 & 0,464 & 0,225 & $2,769 \times 10^{-29}$ \\
\hline$p$ & $p>0,05$ & $\mathrm{p}>0,05$ & $\mathrm{p}>0,05$ & $\mathrm{p}>0,05$ \\
\hline
\end{tabular}

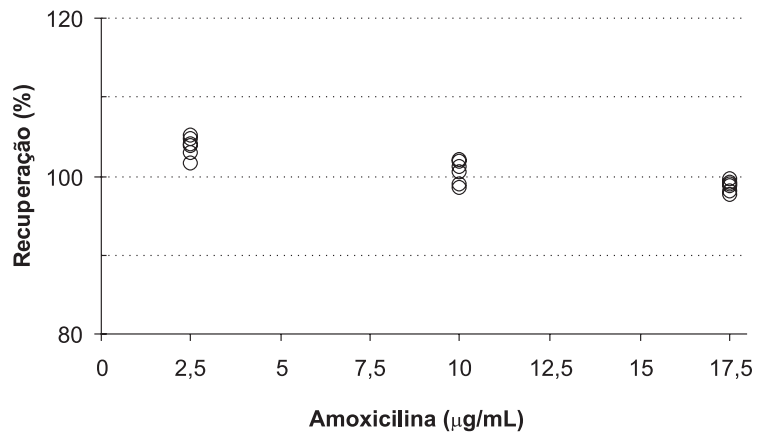

Figura 4. Porcentagens de recuperação individuais obtidas para as seis replicatas de amostras de solução de swab, preparadas nos níveis 2,5; 10,0 e 17,5 $\mu \mathrm{g} / \mathrm{mL}$, analisadas em dois ensaios, sob condições de repetitividade e reprodutibilidade parcial

concentração estudada. Para amostras analisadas no nível de aceitação, considerando a etapa de amostragem, o DPR obtido foi de 4,20\%, enquanto o $\mathrm{DPR}_{\mathrm{R}}$ foi 4,39\%. Foram considerados aceitáveis valores de $D_{P R}$ menores ou iguais a 13,94; 11,31 e 10,40\% para os níveis 2,$5 ; 10,0$ e $17,5 \mu \mathrm{g} / \mathrm{mL}$, respectivamente, correspondentes aos valores estimados pela função de Horwitz. ${ }^{29}$ Para os DPR , os valores máximos aceitáveis foram dois terços dos valores obtidos pela função de Horwitz, ou seja, 9,29; 7,54 e 6,93\%, para 2,5; 10,0 e 17,5 $\mu \mathrm{g} / \mathrm{mL}$, respectivamente. Assim, a precisão do método também foi indicada (Tabela 5).

Tabela 5. Médias de recuperação e desvios padrão relativos, sob condições de repetitividade e reprodutibilidade parcial, obtidos para amostras formuladas

\begin{tabular}{lcccc}
\hline Concentração $(\mu \mathrm{g} / \mathrm{mL})$ & $n$ & $R(\%)$ & $\mathrm{DPR}_{r}(\%)$ & $\mathrm{DPR}_{R}(\%)$ \\
\hline \multirow{4}{*}{ Etapa de amostragem excluída } \\
10,5 & 6 & 103,74 & 0,85 & 1,43 \\
17,5 & 6 & 100,57 & 1,31 & 1,47 \\
\multicolumn{4}{c}{ Etapa de amostragem incluída } \\
10 & 6 & 98,75 & 0,58 & 0,79 \\
\hline
\end{tabular}

$n=$ número de observações; $R=$ média de recuperação; $\mathrm{DPR}_{r}=$ desvio padrão relativo de repetitividade; $\mathrm{DPR}_{R}=$ desvio padrão relativo de reprodutibilidade parcial. Critério de aceitabilidade para $R$, segundo $\mathrm{AOAC}^{24}$ : de 80 a $115 \%$.Critério de aceitabilidade para DPR, segundo Horwitz: ${ }^{29} \leq 9,29 \%$ para $2,5 \mu \mathrm{g} / \mathrm{mL}, \leq 7,54 \%$ para $10,0 \mu \mathrm{g} / \mathrm{mL}$ e $\leq 6,93 \%$ para $17,5 \mu \mathrm{g} / \mathrm{mL}$, correspondente a dois terços do valor obtido pela função de Horwitz. Critério de aceitabilidade para $\mathrm{DPR}_{R}$, segundo Horwitz: $:^{29} \leq 13,94 \%$ para $2,5 \mu \mathrm{g} / \mathrm{mL}, \leq 11,31 \%$ para $10,0 \mu \mathrm{g} / \mathrm{mL}$ e $\leq 10,40 \%$ para 17,5 $\mu \mathrm{g} / \mathrm{mL}$, correspondente aos valores obtidos pela função de Horwitz.

Os limites de detecção e quantificação teóricos estimados corresponderam a 0,133 e $0,442 \mu \mathrm{g} / \mathrm{mL}$, respectivamente. Para amostragens em gabaritos de $25 \mathrm{~cm}^{2}$ estes limites corresponderiam a 3,33 $\mu \mathrm{g}$ e 11,05 sug de amoxicilina. Tais limites sinalizaram a capacidade do 
método para determinação de resíduos de amoxicilina mesmo em casos de critérios de aceitabilidade mais rigorosos, com níveis críticos abaixo da faixa validada neste trabalho.

Na validação de método aplicado ao controle de limpeza para o $\beta$-lactâmico cefmetazol, empregando amostragem por swab, Fukutsu et $a l .{ }^{7}$ avaliaram exatidão e precisão em um único nível de concentração. Resultados médios de recuperação de 62,1; 60,1 e 91,9\% foram obtidos para as diferentes superfícies estudadas. A precisão foi pesquisada somente em condições de repetitividade, com DPR $\mathrm{de}$ $3,6 \%$. Os limites foram estabelecidos como três e dez desvios padrão dos resultados obtidos para replicatas de soluções diluídas do padrão.

\section{Aplicação do método validado}

Todas as amostras analisadas, provenientes do processo de limpeza de três diferentes lotes do pó para suspensão oral de amoxicilina, tiverem resultados conformes, ou seja, abaixo do limite de aceitação estabelecido de $250 \mu \mathrm{g}$ de amoxicilina para o gabarito de $25 \mathrm{~cm}^{2}$. Vinte amostras apresentaram resultados abaixo do limite de quantificação do método. Quatro amostras, provenientes da dosadora de pós, tiveram resultados quantificados entre 19,0 e 34,0 $\mu \mathrm{g}$ de amoxicilina, sendo três delas da hélice de agitação do pó e uma da rosca dosadora.

\section{CONCLUSÃO}

Linearidade, seletividade, exatidão e precisão do método foram comprovadas, com limites de detecção e quantificação suficientemente baixos para determinação de resíduos de amoxicilina em swabs de equipamentos oriundos da produção de pó para suspensão oral. Os parâmetros de desempenho estudados indicaram adequação método para o monitoramento de resíduos de amoxicilina, com controle do processo de limpeza no contexto das BPF.

\section{REFERÊNCIAS}

1. Hardman, J. G.; Limbird, L. E.; Goodman \& Gilmam. As bases Farmacológicas da Terapêutica, 10ª . ed., Editora McGrow-Hill: Rio de Janeiro, 2004.

2. Gil, B.; Newmann, S.; Cleaning validation: a practical approach, Interpharm/CRC Press: Englwood, 1999.

3. Brasil, Ministério da Saúde, Agência Nacional de Vigilância Sanitária (ANVISA); Resolução RDC No210, de 04/08/2003, Regulamento técnico de boas práticas de fabricação de medicamentos.

4. United States Food and Drug Administration (FDA); Guide to inspections validation of cleaning processes, 1993, www.fda.gov/ICECI/Inspections/InspectionGuides/ucm074922.htm, acessada em Junho 2009.

5. Brasil, Ministério da Saúde, Agência Nacional de Vigilância Sanitária (ANVISA); Resolução RE $N^{\circ} 899$, de 29/05/2003, Guia para validação de métodos analíticos e bioanalíticos.

6. Thompson, M.; Ellison, S. L. R.; Wood, R.; Pure Appl. Chem. 2002, 74, 835 .
7. Fukutsu, N.; Konse, T.; Kawasaki, T.; Saito, H.; Nakazawa, H.; J. Pharmaceut. Biomed. Anal. 2006, 41, 599.

8. USP; United States Pharmacopeia, 31 ed., United States Pharmacopeial Convention: Rockville, 2008.

9. Brasil. Ministério da Saúde, Agência Nacional de Vigilância Sanitária (ANVISA), Gerência Geral de Inspeção e Controle de Medicamentos e Produtos (GGIMP); Guias relacionados à garantia de qualidade, ANVISA: Brasília, 2006.

10. Souza, S. V. C.; Tese de Doutorado, Universidade Federal de Minas Gerais, Brasil, 2007.

11. Souza, S. V. C.; Junqueira, R. G.; Anal. Chim. Acta 2005, 552, 25.

12. Meyer, P. C.; Zund, R. E.; Statistical methods in analytical chemistry, John Wiley \& Sons: New York, 1993.

13. Belsley, D. A.; Kuh, E.; Welsch, R. E.; Regression diagnostics: identifying influential data and sources of collinearity, Wiley: New York, 1980.

14. Horwitz, W.; Pure Appl. Chem. 1995, 67, 331.

15. Ryan, T. A.; Joiner, B. L.; Normal probability plots and tests for normality, The State College: Pennsylvania State University, 1976.

16. Levene, H. Em Contributions to probability and statistics; Olkin, I.; Ghurye, S. G.; Hoeffding, W.; Madow, W. G.; Mann, H. B.; eds.; Stanford University Press: Stanford, 1960.

17. Brown, M. B.; Forsythe, A. B.; J. Am. Stat. Assoc. 1974, 69, 364.

18. Durbin, J.; Watson, G. S.; Biometrika 1951, 38, 159.

19. Draper, N.; Smith, H.; Applied regression analysis, Wiley: New York, 1998.

20. Snedecor, G. W.; Cochran, W. G.; Statistical methods, Iowa State University: Ames, 1989.

21. Armitage, P.; Berry, G.; Statistical methods in medical research, Blackwell Science Ltd.: Oxford, 1994.

22. Grubbs, F.; Technometrics 1969, 11, 1.

23. Barret, V.; Lewis, T.; Outliers in statistical data, $3^{\text {rd }}$ ed., John Wiley: New York, 1994.

24. Burke, S.; LC GC 2001, 19.

25. Association of Official Analytical Chemists (AOAC); AOAC Requirements for single laboratory validation of chemical methods, AOAC: Gaithersburg, 2002.

26. International Standards Organization (ISO); Accuracy (trueness and precision) of measurement methods and results, ISO 5725-1, 2, 3, 4, 6, 1994.

27. International Standards Organization (ISO); Accuracy (trueness and precision) of measurement methods and results, ISO 5725-5, 1998.

28. Kuttatharmmakul, S.; Massart, D. L.; Smeyers-Verbeke, J.; Anal. Chim. Acta 1999, 391, 203.

29. Maroto, A.; Riu, J.; Boque, R.; Rius, F. X.; Anal. Chim. Acta 1999, 391, 173.

30. Horwitz, W.; Anal. Chem. 1982, 54, 67A.

31. Alencar, J. R. B.; Ramos, S. V. V.; Machado, L. B.; Oliveira, A. T. C.; Monteiro, D. B.; Medeiros, F. P. M.; Neto, P. J. R.; Rev. Bras. Ciênc. Farm. 2004, 40, 1.

32. Fekete, S.; Fekete, J.; Ganzler, K.; J. Pharmaceut. Biomed. Anal. 2009 , 49,833 . 